\title{
A Comparative Study of Preferential Adsorption in Bromoform (1), Benzene (2), and Polymer (3) Systems by Light Scattering and Density Gradient Ultracentrifugation
}

\author{
J. M. G. Cowie, R. Dey and Jean T. McCrindle \\ Department of Chemistry, University of Stirling, Stirling, Scotland
}

(Received September 26, 1970)

\begin{abstract}
Preferential adsorption in bromoform (1), benzene (2), and polymer (3) systems has been studied by two different methods: sedimentation equilibrium in a density gradient and light scattering. The preferential adsorption parameters were estimated for mixtures containing polystyrene and poly $(\alpha$-methylstyrene) as the polymer component. In both systems, inverse adsorption was observed, in which the poor solvent bromoform is selectively adsorbed by the polymer. This occurred at low bromoform concentrations in the mixture but as the amount of bromoform increased there was a reversion to preferential adsorption of the benzene. An attempt to analyse the data in terms of the thermodynamic interactions indicated that agreement between theory and experimental data could only be obtained if it was assumed that inclusion of a ternary interaction parameter was necessary in the theoretical treatment.

KEY WORDS Preferential Adsorption / Density Gradient / Ultracentrifugation / Light Scattering / Polystyrene / Poly $(\alpha$-methylstyrene) / Apparent Specific Volume /
\end{abstract}

Sedimentation equilibrium in a density gradient has been used to measure small differences in density between two polymer components dissolved in a binary solvent mixture. ${ }^{1,2}$ The sensitivity of the method depends on the gradient formed in the cell and also on the interaction of the polymer with components of the mixture. A recent study of the variation of polymer density with tacticity showed that if there is selective adsorption of one of the liquid components by the polymer the resolution can be greatly reduced or even lost, ${ }^{2}$ and so it is important to be aware of the effects of selective adsorption in such quasi-ternary systems. While it is possible to calculate a preferential adsorption parameter from density gradient studies the data are restricted to the narrow composition range which is required to form a suitable gradient. The density gradient technique relies on the choice of two liquids of densities $\rho_{1}$ and $\rho_{2}$ which are solvents for a polymer of density $\rho_{3}$. If the conditions $\rho_{1}>\rho_{3}>\rho_{2}$ are met, adjustment of the concentrations of the liquid components will produce a mixture whose density closely approximates that of the polymer.
By subjecting this quasi-ternary solution to a force field in an ultracentrifuge, the polymer will collect in a narrow band in the isopycnic region when equilibrium conditions are attained. Calculation of the density of the liquid at this point in the cell allows the density, or apparent specific volume $\nu_{3}{ }^{a}$ of the solvated polymer, to be estimated, but $\nu_{3}{ }^{a}$ for many of the polymers examined in this way differs from that determined by other means. This has been found to be the case in bromoform (1), benzene (2), and polymer (3) systems, where the polymer is either polystyrene or $\operatorname{poly}(\alpha$-methylstyrene). The apparent specific volume for atactic polystyrene was found to be 0.891 and that for atactic poly $(\alpha$-methylstyrene) was 0.844 when measured in this density gradient, whereas pycnometric determination of $\nu_{3}^{a}$ in benzene indicated 0.919 and 0.885 for polystyrene and $\operatorname{poly}(\alpha$-methylstyrene $)$ respectively. ${ }^{2}$

These results imply that either the approximations used in estimating the density of the isopycnic band is insufficiently accurate or there must be extensive solvation of both polymers by the heavy component bromoform. This 
latter conclusion is perhaps rather surprising when a comparison of the solubility parameters shows that benzene should be the solvent most compatible with each polymer. This view is supported by the solution behaviour of the polymers in both solvents; benzene is found to be a thermodynamically good solvent whereas bromoform is rather poor. The possibility of preferential adsorption of bromoform is worth exploring further, however, as other systems have also been reported which exhibit limited selective adsorption of a poor solvent from a binary pair composed of a good and a poor solvent, but these are few in number ${ }^{3,4}$

The binary composition range which proved suitable for the density gradient study is also rather restricted lying in the region of 37.984 to $44.028 \mathrm{~g}$ bromoform $/ 100 \mathrm{~cm}^{3}$ of solution and a more extensive examination of preferential adsorption in ternary systems can be made by means of light scattering which will provide information over the entire composition range. Preferential adsorption parameters have been derived from data obtained using both light scattering and density gradient techniques and an attempt to relate these to the thermodynamic interaction parameters has been made.

\section{EXPERIMENTAL METHODS}

\section{Density Gradient Ultracentrifugation}

Apparent specific volumes $\nu_{3}{ }^{a}$ were determined in gradients formed by bromoform (1), and benzene (2) mixtures as detailed previously. ${ }^{2}$ For poly $(\alpha$-methylstyrene $)$ a suitable gradient was formed from $40.014 \mathrm{~g}$ bromoform in 100 $\mathrm{cm}^{3}$ of solution, while $37.984 \mathrm{~g}$ bromoform $/ 100$ $\mathrm{cm}^{3}$ solution was found to be more suitable for polystyrene. Measurements were made in a Spinco model E ultracentrifuge equipped with schlieren optics and equilibrium was established in 40 to $72 \mathrm{hr}$ at rotor speeds of $33450 \mathrm{rpm}$.

\section{Light Scattering}

Light scattering measurements were made in a SOFICA instrument at $25^{\circ} \mathrm{C}$ using vertically polarized blue light of wave length $436 \mathrm{~nm}$. Solutions were contained in cells fitted with teflon stoppers to ensure that differential evaporation of the solvent components was minimized during measurements. The samples used were narrow molecular weight distribution polymers prepared by anionic polymerization techniques. Solutions were clarified by double filtration through grade 5 sintered glass filters. Molecular weights were calculated using a value of $R_{90}=49 \times 10^{-6}$ for the Rayleigh ratio of pure benzene.

The refractive index increment $(\mathrm{d} n / \mathrm{d} c)$ of the polymers in each mixture was determined in a Brice-Phoenix differential refractometer at $25^{\circ} \mathrm{C}$ using blue light. Care was taken to ensure that the composition of the solvent remained constant throughout the measurement.

\section{RESULTS}

\section{Apparent Specific Volumes}

Calculation of $\nu_{3}{ }^{a}$ from data obtained in a density gradient involves estimation of the volume fraction of solvent components in the isopycnic region. The density of the polymer is then taken to be an equivalent value. Results obtained in this way are shown in Table I where they can be compared with values measured at the same temperature in benzene using a pycnometer. Also shown are values of the unsolvated polymers.

The differences are quite significant, but as the high rotor speeds in the ultracentrifugation generate a considerable pressure gradient in a cell this effect of pressure on the density should be examined. The pressure $P(x)$ at any point $x$ in the ultracentrifuge cell can be approximated from

$$
P(x)=\frac{\omega^{2} \rho_{m}\left(x^{2}-x_{0}{ }^{2}\right)}{2}
$$

where $\omega$ is the angular velocity in radians/sec, $\rho_{m}$ is the density of the mixture, $x_{0}$ and $x$ are the distance of the meniscus and point in question in the solution from the centre of rotation. The influence of an increased pressure on the density of the medium can then be estimated if the compressibility $\kappa$ of the solution is known and substituted in

$$
\rho_{m}=\rho_{m}{ }^{0}(1+\kappa P)
$$

where $\rho_{m}{ }^{0}$ is the density at 1 atmosphere and $\rho_{m}$ the corrected density at pressure $\boldsymbol{P}$.

As the major component in the mixture was 
Table I. Apparent specific volumes for polystyrene and poly ( $\alpha$-methylstyrene) measured by pycnometry, and a density gradient method corrected and uncorrected for pressure effets

\begin{tabular}{lcccc}
\hline Polymer & $\begin{array}{c}\nu_{3} a, \mathrm{~cm}^{3} / \mathrm{g} \\
\text { (density gradient) }\end{array}$ & $\begin{array}{c}\nu_{3} a, \mathrm{~cm}^{3} / \mathrm{g} \\
\left(\begin{array}{l}\text { D.G. corrected } \\
\text { for pressure) }\end{array}\right.\end{array}$ & $\begin{array}{c}\nu_{3} a, \mathrm{~cm}^{3} / \mathrm{g} \\
(\text { pycnometer })\end{array}$ & $\nu_{3}^{\mathrm{a}}, \mathrm{cm}^{3} / \mathrm{g}$ \\
\hline Polystyrene & 0.891 & 0.886 & 0.919 & 0.956 \\
Poly $(\alpha$-methylstyrene) & 0.844 & 0.840 & 0.885 & 0.932 \\
\hline
\end{tabular}

a Unsolvated polymer (reciprocal to bulk density).

benzene, $\rho_{m}$ was estimated using a value of $\kappa=$ $0.097 \times 10^{-9} \mathrm{~cm}^{2} / \mathrm{dyn}^{5}$ for benzene, corresponding to a pressure of about $0.53 \times 10^{8} \mathrm{dyn} / \mathrm{cm}^{2}$ in the isopycnic region. The pressure corrected $\nu_{3}{ }^{a}$ for both polymers is less than the uncorrected values and this suggests that the observed differences arise predominantly from selective adsorption of the heavier component bromoform.

Preferential Adsorption Parameter $\Gamma^{\prime}$

While observed differences in $\nu_{3}{ }^{a}$ can suggest preferential adsorption of a solvent component, a semiquantitative estimate can be obtained by calculating the preferential adsorption parameter $\Gamma^{\prime}$. For a quasi-ternary system, the parameter calculated from density gradient measurements is defined on a weight basis by ${ }^{6}$

$$
\Gamma^{\prime}=\frac{M}{M_{3}} \cdot\left(\frac{\partial m}{\partial m_{3}}\right)_{\mu}
$$

where $M$ and $M_{3}$ are the molecular weights of the adsorbed component and the polymer respectively and $m, m_{3}$ are the corresponding concentrations expressed in molalities at constant chemical potential $\mu$. It has been shown ${ }^{6}$ that the parameter $\Gamma^{\prime}$ can be found using the relation

$$
\Gamma^{\prime}=\frac{\left(1-\rho_{m}{ }^{0} \bar{\nu}_{3}\right)}{\left(\rho_{m}{ }^{0} \bar{\nu}-1\right)}
$$

where $\bar{\nu}_{3}$ and $\bar{\nu}$ are the partial specific volumes of the polymer and solvating species and $\rho_{m}{ }^{0}$ is the calculated density of the solvent mixture at the point in the cell where the polymer collects at equilibrium. Eq 4 can be recast if pressure effects are taken into consideration and becomes

$$
\Gamma^{\prime}=\frac{\left[1-\bar{\nu}_{3} \rho m^{0}(1+\kappa P)\right]}{\left[\bar{\nu} \rho m^{0}(1+\kappa P)-1\right]}
$$

The adsorption parameter $\Gamma^{\prime}$ is expressed as the weight of solvating species in $\mathrm{g}$ adsorbed $/ \mathrm{g}$ of polymer and is a measure of the weight of the solvent which would have to be transferred with unit weight of polymer when this is added to a very large volume of solution, if the chemical potential is to remain constant.

Substitution of data in eq 5, accepting that bromoform is the component which is preferentially adsorbed, leads to $\Gamma^{\prime}$ (polystyrene)= 0.059 at $\phi_{1}=0.124$ and $\Gamma^{\prime}$ (poly $(\alpha$-methylstyrene) $)=0.092$ at $\phi_{1}=0.1555$, where $\phi_{1}$ is the volume fraction of bromoform in the mixture.

\section{Light Scattering-Preferential Adsorption}

Confirmation that bromoform is preferentially adsorbed in the composition range employed in the density gradient work, is readily obtained from measurements of the apparent molecular weight $M_{3}^{\text {app }}$. The corresponding adsorption parameter is denoted as $\lambda^{*}$ and defined by ${ }^{7}$

$$
\lambda^{*}=\frac{V_{1}}{V_{3}} \bar{\nu}_{3} \phi_{2}\left(\frac{\partial m_{1}}{\partial m_{3}}\right)_{\mu_{1}}
$$

when it is assumed that component (1) has been adsorbed, and $V$ is the molar volume. A more useful form for $\lambda^{*}$ is

$$
\lambda^{*}=\left[\left(\frac{M_{3 \mathrm{app}}}{M_{3}}\right)^{1 / 2}-1\right] \frac{(\mathrm{d} n / \mathrm{d} c)}{\left(\mathrm{d} n_{\mathrm{bm}} / \mathrm{d} \phi_{1}\right)}
$$

The correct molecular weight of the polymer $M_{3}$ can be measured in a single solvent and comparison can then be made with the apparent molecular weight $M^{\text {app }}$ measured in the solvent mixture, $(\mathrm{d} n / \mathrm{d} c)$ is the refractive index increment of the quasi-ternary system measured directly in the refractometer and $\left(\mathrm{d} n_{\mathrm{bm}} / \mathrm{d} \phi_{1}\right)$ is the refractive index gradient of the binary liquid mixture expressed as a function of the volume fraction of bromoform. This is approximately +0.10 . In the systems used here $n_{3}=n_{1}>n_{2}$. 


\section{Comparative Study of Preferential Adsorption}

Table II. Apparent molecular weights, refractive index increments, and preferential adsorption parameters for various compositions of the systems bromoform

(1), benzene (2), and polymer (3)

\begin{tabular}{|c|c|c|c|c|c|c|}
\hline \multirow{2}{*}{$\begin{array}{c}\text { Solvent } \\
\text { composition, } \\
\phi_{1}\end{array}$} & \multicolumn{3}{|c|}{ Polystyrene } & \multicolumn{3}{|c|}{$\operatorname{Poly}(\alpha$-methylstyrene $)$} \\
\hline & $(\mathrm{d} n / \mathrm{d} c)$ & $10^{5} \times \bar{M}_{w}$ & $\lambda^{*}$ & $(\mathrm{~d} n / \mathrm{d} c)$ & $10^{5} \times \bar{M}_{w}$ & $\lambda^{*}$ \\
\hline 0.00 & 0.111 & 1.27 & - & 0.128 & 3.93 & - \\
\hline 0.05 & 0.101 & 1.32 & +0.020 & 0.124 & 4.06 & +0.022 \\
\hline 0.10 & 0.096 & $1.32_{5}$ & +0.021 & 0.120 & 4.85 & +0.130 \\
\hline 0.15 & 0.099 & 1.26 & -0.004 & 0.118 & 4.27 & +0.052 \\
\hline 0.25 & 0.083 & 1.19 & -0.027 & 0.109 & 3.84 & -0.013 \\
\hline 0.50 & 0.074 & 1.00 & -0.084 & 0.079 & 2.72 & -0.134 \\
\hline 0.75 & 0.036 & $0.70_{6}$ & -0.092 & 0.051 & 2.61 & -0.094 \\
\hline
\end{tabular}

As the refractive index of bromoform is closer to that of each polymer than the refractive index of benzene, the parameter $\lambda^{*}$ is positive when bromoform is adsorbed and negative when benzene is adsorbed preferentially. Detailed results, given in Table II, show that there is preferential adsorption of bromoform (1) when the concentration of this component in the mixture is low. As $\phi_{1}$ increases beyond $0.15-$ 0.20 in both systems the adsorption of bromoform quickly decreases and is replaced by a selective adsorption of benzene into the solvation hull around the polymer. Preferential adsorption effects are not excessive in either system but $\lambda^{*}$ appears more pronounced when $\operatorname{poly}(\alpha$-methylstyrene $)$ is the polymer.

\section{Thermodynamic Analysis}

Attempts to describe the thermodynamic interactions in quasi-ternary systems in terms of binary $\chi_{i j}$ and ternary $\chi_{i j k}$ interaction parameters usually lead to expressions relating these to the extent of the preferential adsorption in the mixture. One such relationship, which was proposed by Read, ${ }^{7}$ has the form
Table III. Binary interaction parameters for bromoform (1), benzene (2), and polymer (3) systems

\begin{tabular}{lll}
\hline Polymer & $\chi_{13}$ & $\chi_{23}$ \\
\hline Polystyrene & 0.72 & 0.34 \\
Poly $(\alpha$-methylstyrene $)$ & 0.63 & 0.36 \\
\hline
\end{tabular}

tivity data are required to provide accurate $\chi_{i j}$ parameters for the binary liquid systems and these are not readily available in the literature. An alternative but less accurate procedure, is to estimate $\chi_{i j}$ from the component solubility parameters $\delta$ using

$$
\chi_{i j}=\frac{V_{M}}{R T}\left(\delta_{i}-\delta_{j}\right)^{2}
$$

where $V_{M}$ is the molar volume of the mixture. If the liquids and polymers are essentially non polar, this can provide a useful approximation. The binary interaction parameters shown in Table III were calculated from eq. 9.

One would expect the $\chi_{12}$ parameter to have the same value for both systems but a value

$$
\lambda^{*}=\frac{\bar{\nu}_{3} \phi_{1} \phi_{2}\left[\left(\chi_{12}-\chi_{123}\right)\left(\phi_{2}-\phi_{1}\right) l^{-1}+\chi_{23}-l^{-1} \chi_{13}+\left(l^{-1}-1\right)\right]}{\phi_{1}+l^{-1} \phi_{2}-2 \chi_{12} l^{-1} \phi_{1} \phi_{2}}
$$

where $l$ is the ratio of molar volumes of the two liquid components $\left(V_{1} / V_{2}\right)$. It has been shown that in some systems inclusion of the ternary interaction parameter is unnecssary, ${ }^{4,8}$ but in others $\chi_{i j k}$ must be used if there is to be any semblance of agreement between the experimental data and theory. ${ }^{2}$ For a critical assessment of relations such as eq 8 , good ac- which is composition dependent would be necessary if the shape of the $\lambda^{*}$ against $\phi_{1}$ curve is to be matched using eq 8 . Zivny, et al. ${ }^{3}$ found that $\chi_{12}$ in the benzene-methanol system varied quite strongly with the composition of the mixture, decreasing as the methanol concentration increased.

Unfortunately the data for both systems could 


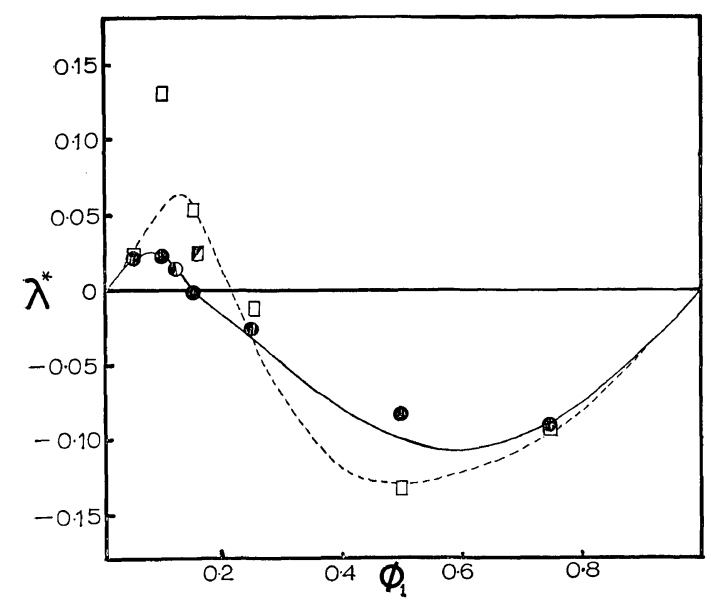

Figure 1. Dependence of $\lambda^{*}$ on the volume fraction of bromoform $\phi_{1}$ for polystyrene ( $-\mathbf{0}-$ ), and $\operatorname{poly}(\alpha$-methylstyrene) (--- $\square---)$. Solid and broken curves are derived from eq 8 . Converted values of $\lambda^{*}$ from ultracentrifugation data: - - , for polystyrene and --- for poly ( $\alpha$-methylstyrene).

not be represented using a single variable $\chi_{12}$ and approximate agreement between theory and experiment was obtained only when the $\chi_{123}$ factor was assumed to be significant. This modifies the $\chi_{12}$ value and the curves in Figure 1 were constructed from eq 8 by choosing a variable $\left(\chi_{12}-\chi_{123}\right)$ factor for each system. Moderately good agreement was obtained when this factor was allowed to decrease from unity to zero for the polystyrene system and unity to 0.2 for the poly $(\alpha$-methylstyrene $)$ system as the concentration of bromoform in the mixtures increased. Calculation of a $\chi_{12}$ parameter from eq 9 gave a value of $\sim 0.7$. This is smaller than required and unless a negative $\chi_{123}$ is assumed at low bromoform concentrations the curves will not fit the data. Although a negative $\chi_{123}$ is not an impossibility, it is a rather questionable assumption which is difficult to describe in physical terms, and it is more reasonable to consider that $\chi_{12}$ has a minimum value of unity and that eq 9 underestimates the magnitude of this parameter. A curve fit can now be forced using this means but until good experimental values for $\chi_{i j}$ and $\chi_{i j k}$ parameters can be obtained the validity of expressions such as eq 8 cannot be confirmed.

\section{DISCUSSION}

The preferential adsorption parameters calculated from the two different experimental methods can be related by

$$
\lambda^{*}=\Gamma^{\prime}\left(\frac{\phi_{2}}{\rho_{1}}\right)
$$

The sedimentation equilibrium parameters $\Gamma^{\prime}$ were converted into $\lambda^{*}$ and plotted in Figure 1 for comparison. Reasonable agreement was found, though the calculation of $\lambda^{*}$ from light scattering apparently results in somewhat larger values than those measured using the density gradient method. As $\lambda^{*}$ obtained from light scattering is essentially a measure of preferential adsorption at infinite dilution, whereas $\Gamma^{\prime}$ was measured at a fixed concentration of $0.0014 \mathrm{~g} /$ $\mathrm{cm}^{3}$, differences might be expected between $\lambda^{*}$ and $\Gamma^{\prime}$ but these should be quite small in this range.

The preferential adsorption of the poorer solvent component bromoform was detected below $\phi_{1}=0.2$ in each system by both methods of measurement. This effect, which is sometimes called "negative" adsorption, has been detected in the benzene/methanol/poly(methyl methylacrylate) system ${ }^{3}$ and has been theoretically predicted for the benzene/cyclohexane/ polystyrene system $^{7}$ without actually being found experimentally. It is perhaps rather misleading to term it "negative" adsorption as the sign of the preferential adsorption parameter depends on the convention adopted, ${ }^{9}$ and it might be better to term it "inverse" adsorption.

Suh and Liou $^{10}$ have suggested that for systems in which a good solvent, component (1), has a lower molar volume than a poor solvent, component (2), and where the difference in the solubility parameters of (2) and the polymer (3) exceeds $2.0\left(\mathrm{cal} / \mathrm{cm}^{3}\right)^{1 / 2}$, then selective adsorption of component (2) can be expected at low values of $\phi_{2}$. While such conditions lead to inverse adsorption in solvent precipitant mixtures, it is unnecessary to adhere rigidly to these criteria in order to observe this phenomenon, but, without accurate $\chi_{i j}$ parameters the underlying reasons can only be speculated upon. It is possible that a major 


\section{Comparative Study of Preferential Adsorption}

factor may be the liquid-liquid interaction embodied in $\chi_{12}$. As mentioned earlier the benzene/methanol $\chi_{12}$ varies with composition and at low methanol concentrations the mixtures showed marked incompatibility. If addition of small quantities of one liquid to a second is not a process which is energetically favourable, then the liquid in low concentration may find that the domain of a dissolved polymer is initially more compatible and tend to accumulate in this region even though it is a poorer solvent for that polymer. As the concentration of the poor solvent increases in the mixture, the cohesive energy of the bulk of the solvent becomes progressively more compatible with the poor solvent which is then replaced by the good solvent in the vicinity of the polymer. This is likely to be an oversimplification in certain systems, especially those in which the ternary interaction parameter cannot be neglected. When ternary interactions have to be considered, the thermodynamic analysis becomes increasingly complex.

\section{REFERENCES}

1. R. Buchdahl, H.A. Ende, and L. H. Peebles, I. Polym. Sci., Part C, 1, 143, 153 (1963).

2. J. M. G. Cowie and P. M. Toporowski, Eur. Polym. J., 5, 493 (1969).

3. A. Zivny, J. Pouchly, and K. Sole, Collect. Czech. Chem. Commun., 32, 2753 (1967).

4. C. Strazielle and H. Benoit, J. Chim. Phys., 58, 675 (1961).

5. R. L. Baldwin and K. E. van Holde, Advan. Polym. Sci., 1, 451 (1960).

6. J. Vinograd and J. E. Hearst, Prog. Chem. Org. Nat. Prod., 20, 372 (1962).

7. B. E. Read, Trans. Faraday Soc., 56, 382 (1960).

8. J. M. G. Cowie and S. Bywater, J. Macromol. Chem., 1, 581 (1966).

9. J. M. G. Cowie, J. Pure Appl. Chem., in press.

10. K. W. Suh and D. W. Liou, J. Polym. Sci., Part A-2, 6, 813 (1968). 